\title{
Practical Numerical Simulations of Two-Phase Flow and Heat Transfer Phenomena in a Thermosyphon for Design and Development
}

\author{
Zheshu $\mathrm{Ma}^{1}$, Ali Turan ${ }^{2}$, and Shengmin Guo ${ }^{2}$ \\ ${ }^{1}$ Department of Power Engineering, Jiangsu University of Science and Technology, \\ Zhenjiang, 212003, China \\ ${ }^{2}$ School of Mechanical, Aerospace and Civil Engineering, The University of Manchester, \\ Manchester, M60 1QD, UK \\ mazheshu@126.com, A.Turan@manchester.ac.uk, sguo2@lsu.edu
}

\begin{abstract}
Two-phase closed thermosyphons are widely-used in various energy intensive industries including chemical, transportation and other industries for their inherently high heat transfer efficiency, simple construction and reliable operational performance. However, the performance parameters of two-phase closed thermosyphons including the distribution of internal pressure, steam and liquid phase mass fractions, velocity and wall temperatures are obtained primarily via experimental investigations. In this paper numerical methods are discussed and a two-fluid model is employed to describe the two-phase flow and heat transfer processes in a two-phase closed thermosyphon. The IPSA (Inter Phase Slip Algorithm) algorithm is employed to solve the coupled interactions of steam and liquid phases along the phase interface. Flow patterns and distribution of parameters under different conditions are predicted with numerical results agreeing well for the most part with experimental results. Thus, the numerical method and solution procedures are claimed to be of practical utility and can in essence be used profitably to simulate flow and heat transfer phenomena in thermosyphons and other types of heat pipes.
\end{abstract}

Keywords: IPSA algorithm, two-phase flow, numerical simulation, thermosyphon.

\section{Introduction}

With the integration of electronic circuits and greatly increasing power capacity of power appliances, cooling methods with improved efficiency that can provide suitable thermal environments for electronic equipments are required, as electronic components are constantly being miniaturized in size. Today, electronic cooling technologies have become one of the key considerations in design and operation of electronic equipment [1-3]. State-of-the-art cooling technologies and developments related to high power electronic devices include a variety of processes ranging from phase-change cooling, forced convection cooling, natural convection cooling and micro heat exchanger cooling [4]. Heat pipe electronic cooling is a kind of phase-change cooling technology 
possessing several striking advantages, i.e., a large equivalent thermal conductance, excellent packaging flexibility, passive operation and high reliability [4].

Heat pipes including two-phase closed thermosyphons are a promising phasechange electronic cooling technology for the various advantages offered including high heat transfer efficiency, simple construction and reliable operational performance. In order to analyze their running performance and to further design more efficient and reliable two-phase closed thermosyphons, designers and researchers must possess a sound understanding of the internal two phase flow details and heat transfer characteristics in a thermosyphon, in particular looking at the subcooled boiling process for the liquid including vapor release from the saturated liquid, vapor condensation back to the liquid, upward flow of vapor and downward flow of condensed liquid, and different flow regimes varying according to internal flow characteristics as regulated by the operational conditions of thermosyphons. Since the inherent internal flow and heat transfer details are very complicated, the performance parameters of two-phase closed thermosyphons including the distribution of internal pressure, steam and liquid phase mass fractions, velocity and wall temperatures are currently primarily obtained and even designed by exclusively devising appropriate experimental studies.

Computational simulation procedures employing advanced engineering mathematical and physical models via high performance computers have been playing more and more important roles in most engineering fields for the inherent advantages offered including efficiency and accuracy, especially for cases where measurements are impractical. Specifically, regarding the numerical simulation of two phase flow phenomena within heat pipes, state-of-the-art solutions initially analyze the internal vapor-liquid two phase flow regimes, to subsequently identify flow characteristics for the different flow zones and finally appropriate mathematical and physical models are numerically solved.

In this paper, a specific numerical method including a two-fluid model is employed to describe the two-phase flow and heat transfer processes in a two-phase closed thermosyphon. Widely used IPSA algorithm [5-7, 9] is employed to solve the coupled interaction of steam and liquid phases along the phase interface. Flow patterns and distribution of parameters under different conditions are predicted. Numerical results agree well with experimental results.

\section{Mathematical Model and Numerical Method}

To numerically simulate the complex heat transfer and two-phase flow phenomena in the thermosyphon configuration studied, a two-fluid model is employed. The twofluid model provides an effective means of representing the coexistence of laminar and turbulent flows. It considers the system to be composed of two fluids that coexist simultaneously in time and space but possess different volume fractions and can naturally reflect the exchange of mass, momentum and energy between the two fluids and is well suited to describe the whole computational domain.

When the two-fluid model is applied to predict the thermo-fluid details within the thermosyphon, the following assumptions are naturally made to achieve a workable numerical model: (1)The heat conduction resistance between the outer surface of the 
pipe and the inner wall of the pipe is neglected; (2)The thermodynamic properties of the working vapor and liquid are assumed to be constant ,i.e., vapor and liquid phases are saturated; (3)Vapor and liquid flows are laminar and incompressible; (4)The evaporation and condensation processes only occur at the vapor-liquid interface.

The basic governing equations in two dimensions describing the heat transfer and flow of the vapor phase within the studied thermosyphon are provided in Eqs (1) to (4).

Continuity equation for the vapor:

$$
\frac{\partial}{\partial x}\left(R_{1} \rho_{1} u_{1}\right)+\frac{\partial}{\partial y}\left(R_{1} \rho_{1} v_{1}\right)=\dot{m}_{21}
$$

Momentum equations for the vapor:

$$
\begin{gathered}
\frac{\partial}{\partial x}\left(R_{1} \rho_{1} u_{1}^{2}\right)+\frac{\partial}{\partial y}\left(R_{1} \rho_{1} u_{1} v_{1}\right)=-R_{1} \frac{\partial p}{\partial x}+R_{1} \mu_{1} \frac{\partial^{2} u_{1}}{\partial y^{2}}+\dot{m}_{21}\left|u_{1}-u_{2}\right|-F_{21}-R_{1} \rho_{1} g \\
\frac{\partial}{\partial x}\left(R_{1} \rho_{1} u_{1} v_{1}\right)+\frac{\partial}{\partial y}\left(R_{1} \rho_{1} v_{1}^{2}\right)=-R_{1} \frac{\partial p}{\partial y}+R_{1} \mu_{1} \frac{\partial^{2} v_{1}}{\partial x^{2}}+\dot{m}_{21}\left|v_{1}-v_{2}\right|
\end{gathered}
$$

Energy equation for the vapor:

$$
\frac{\partial}{\partial x}\left(R_{1} \rho_{1} u_{1} h_{1}\right)+\frac{\partial}{\partial y}\left(R_{1} \rho_{1} v_{1} h_{1}\right)=R_{1} \frac{\mu_{1}}{\operatorname{Pr}_{1}}\left(\frac{\partial^{2} h_{1}}{\partial y^{2}}\right)+\dot{m}_{21}\left|h_{1}-h_{2}\right|+J_{c}
$$

The basic governing equations in two dimensions describing the heat transfer and the flow of the liquid phase within the thermosyphon are given in Equations (5) to (8).

Continuity equation for the liquid:

$$
\frac{\partial}{\partial x}\left(R_{2} \rho_{2} u_{2}\right)+\frac{\partial}{\partial y}\left(R_{2} \rho_{2} v_{2}\right)=-\dot{m}_{21}
$$

Momentum equations for the liquid:

$$
\begin{gathered}
\frac{\partial}{\partial x}\left(R_{2} \rho_{2} u_{2}^{2}\right)+\frac{\partial}{\partial y}\left(R_{2} \rho_{2} u_{2} v_{2}\right)=-R_{2} \frac{\partial p}{\partial x}+R_{2} \mu_{2} \frac{\partial^{2} u_{2}}{\partial y^{2}}-\dot{m}_{21}\left|u_{1}-u_{2}\right|+F_{21}-R_{2} \rho_{2} g \\
\frac{\partial}{\partial x}\left(R_{2} \rho_{2} u_{2} v_{2}\right)+\frac{\partial}{\partial y}\left(R_{2} \rho_{2} v_{2}^{2}\right)=-R_{2} \frac{\partial p}{\partial y}+R_{2} \mu_{2} \frac{\partial^{2} v_{2}}{\partial x^{2}}-\dot{m}_{21}\left|v_{1}-v_{2}\right|
\end{gathered}
$$

Energy equation for the liquid:

$$
\frac{\partial}{\partial x}\left(R_{2} \rho_{2} u_{2} h_{2}\right)+\frac{\partial}{\partial y}\left(R_{2} \rho_{2} v_{2} h_{2}\right)=R_{2} \frac{\mu_{2}}{\operatorname{Pr}_{2}}\left(\frac{\partial^{2} h_{2}}{\partial y^{2}}\right)-\dot{m}_{21}\left|h_{1}-h_{2}\right|-J_{c}+\frac{C_{2}^{w}}{A_{2}} \dot{Q}
$$


In equations (1) to (8), $\mathrm{x}$ and $\mathrm{y}$ are axial coordinate and diametrical coordinate, respectively; $R_{1}$ and $R_{2}$ are the volume fractions of vapor phase and liquid phase respectively; $\dot{m}_{21}$ is the interphase mass transfer rate between the vapor and liquid, $\left(\mathrm{kg} /\left(\mathrm{m}^{3} . \mathrm{s}\right)\right) . \quad u_{1}$ and $v_{1}$ are axial and radial velocity of the vapor phase respectively, $(\mathrm{m} / \mathrm{s})$ while $u_{2}$ and $v_{2}$ denote the axial and radial velocity of the liquid, $(\mathrm{m} / \mathrm{s}) . \quad \mu_{1}$ and $\mu_{2}$ are the dynamic viscosity coefficients for the vapor and liquid (N.s $/ \mathrm{m}^{2}$ ), while $h_{1}$ and $h_{2}$ signify the specific enthalpies of the vapor and liquid, respectively $(\mathrm{kJ} / \mathrm{kg}) . \rho_{1}$ and $\rho_{2}$ are the densities of the vapor and liquid, $\left(\mathrm{kg} / \mathrm{m}^{3}\right) . \quad F_{21}$ refers to the interphase friction between the vapor and liquid on the phase interface, $(\mathrm{N}) . J_{c}$ is heat transfer rate between the vapor and liquid (W/s) while $\dot{Q}$ refers to the heat addition to the thermosyphon, (W/s) with $C_{2}^{w}$ denoting the length of the wetted perimeter, $(\mathrm{m})$. Finally, $A_{2}$ is the contact area of the liquid phase with the pipe wall, $\left(\mathrm{m}^{2}\right)$ and $\operatorname{Pr}_{1}$ and $\mathrm{Pr}_{2}$ are the Prandtl numbers for the vapor and liquid phases respectively.

During the numerical calculation, $R_{1}$ and $R_{2}$ must satisfy Eq. (9).

$$
R_{1}+R_{2}=1.0
$$

The mass transfer rate between the vapor and liquid phases, $\dot{m}_{21}$, can be described as given by Eq. (10).

$$
\dot{m}_{21}=\frac{J_{c}}{h_{f g}}
$$

where $h_{f g}$ is the vaporization latent heat of the working liquid, in $\mathrm{kJ} / \mathrm{kg}$.

Heat transfer rate between vapor phase and liquid phase, $J_{c}$, is defined as given by the sum of vapor phase heat transfer rate $J_{c 1}$ and the liquid phase heat transfer rate $J_{c 2}$.

$$
\begin{gathered}
J_{c}=J_{c 1}+J_{c 2} \\
J_{c 1}=c_{2} R_{1} R_{2} \rho_{2}\left(h_{1}-h_{1, s}\right) \\
J_{c 2}=c_{3} R_{1} R_{2} \rho_{2}\left(h_{2}-h_{2, s}\right)
\end{gathered}
$$

Friction force between the vapor phase and the liquid phase, $F_{21}$, can be written as in Eq. (14).

$$
F_{21}=c_{1} R_{1} R_{2} \rho_{2}\left(u_{2}-u_{1}\right)
$$

Here $c_{1}, c_{2}$ and $c_{3}$ in Eq.(11)-Eq.(14) are different empirical constants, while $h_{1, s}$ and $h_{2, s}$ are the specific enthalpy of the vapor phase and the liquid phase along the phase interface respectively. 
A conservative finite-volume method with a staggered variable arrangement [7] is used to solve the nonlinear, coupled system of partial differential equations outlined in the previous section. The solution domain is subdivided into a large number of differential control volumes for the scalar variables and velocities. Nodal values of the scalar quantities are located at the geometric centres of their control volumes while the velocities are stored on the scalar control volume faces. Temporal derivatives are discretized by a fully implicit first order backward Euler scheme. Regarding the present cases investigated, the equations arising from the discretization procedure can be assembled in the form of a quasi-tridiagonal matrix which is solved via an Alternating Direction Implicit (ADI) version of the direct Thomas-algorithm. The discretized equations are numerically coupled and the solution is henceforth obtained by employing the widely available iterative and segregated IPSA-solver methodology developed by Spalding [7]. At each time step, the volume fractions and the velocity fields are initially predicted using the previous time step pressure field, which are then corrected via the solution of a "pressure correction equation" derived from the overall mass conservation requirement. The values of pressure and velocities are subsequently updated to satisfy mass-conservation to yield converged values to be used as the initial field for recalculating the flow variables at the next level of iteration. The IPSAprocedure is iteratively repeated until a pre-set convergence criterion is met. As an additional convergence enhancement, the Partial Elimination Algorithm (PEA) is also employed. Convergence of the method is generally declared as the sum of the absolute residual norms falls below 0.001 for all the variables.

\section{Results and Discussions}

The thermosyphon studied in this paper employed water as the working media and the heat pipe was made of bronze [10]. The thermosyphon was 1.1 meters long and its inner diameter and wall thickness are $25 \mathrm{~mm}$ and $2.5 \mathrm{~mm}$ respectively. According to the relevant operational characteristics as validated upon performing appropriate measurements, the lengths of evaporator and condenser sections, including the isothermal region are given as: the axial length of the evaporator section is about $510 \mathrm{~mm}$, while the axial length of condenser section is specified to be $420 \mathrm{~mm}$. Finally, the axial length of isothermal section is assumed to be $170 \mathrm{~mm}$. Heat addition density is specified as $10 \sim 60 \mathrm{~kW} / \mathrm{m}^{2}$.

Fig.1 indicates the average wall temperature in the evaporator section under different heat addition densities. With the increase of heat addition density which signifies a higher total heat transfer quantity, the average wall temperature in evaporator section increases. From Fig.1, one can see the computational results agree well with the experimental results. The computational field also suggests a linear relationship regarding the variation of the average temperature in evaporator section versus the heat addition density. The experimental results which deviate somewhat from the predicted computational line are suggestive of external influences brought on by the particular experimental procedures, the test setup and other operational and measurement inaccuracies. Such factors arise mainly from practical heat transfer considerations that could not be strictly prevented using the current experimental setup to provide for rigorous adiabaticity in the adiabatic section of the working hardware. 


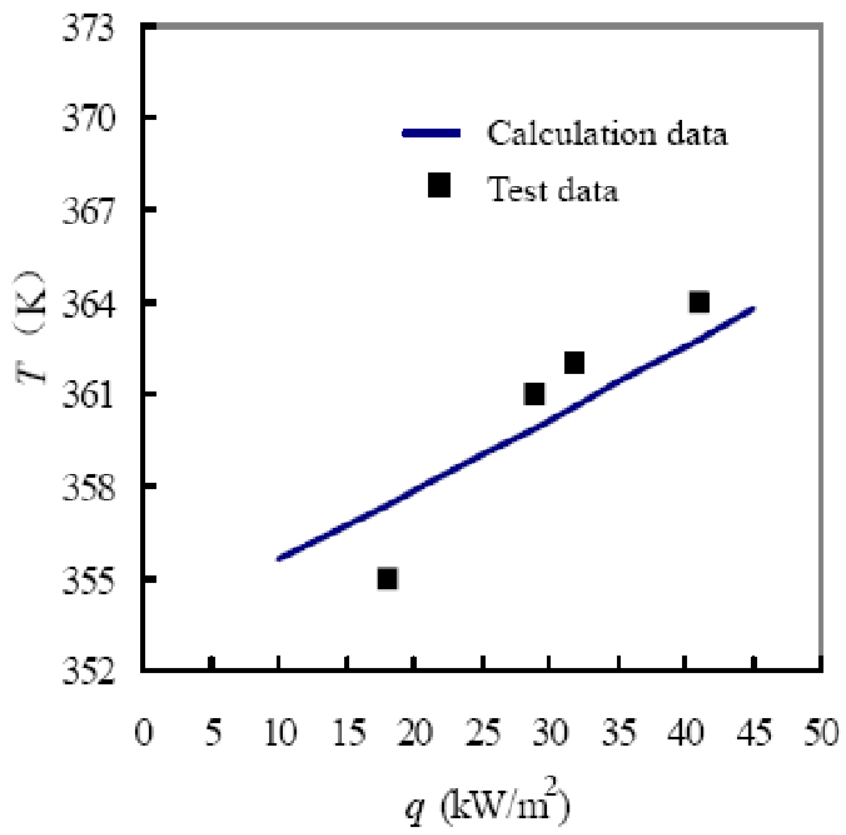

Fig. 1. Wall temperature in the evaporator section varying with heat addition density

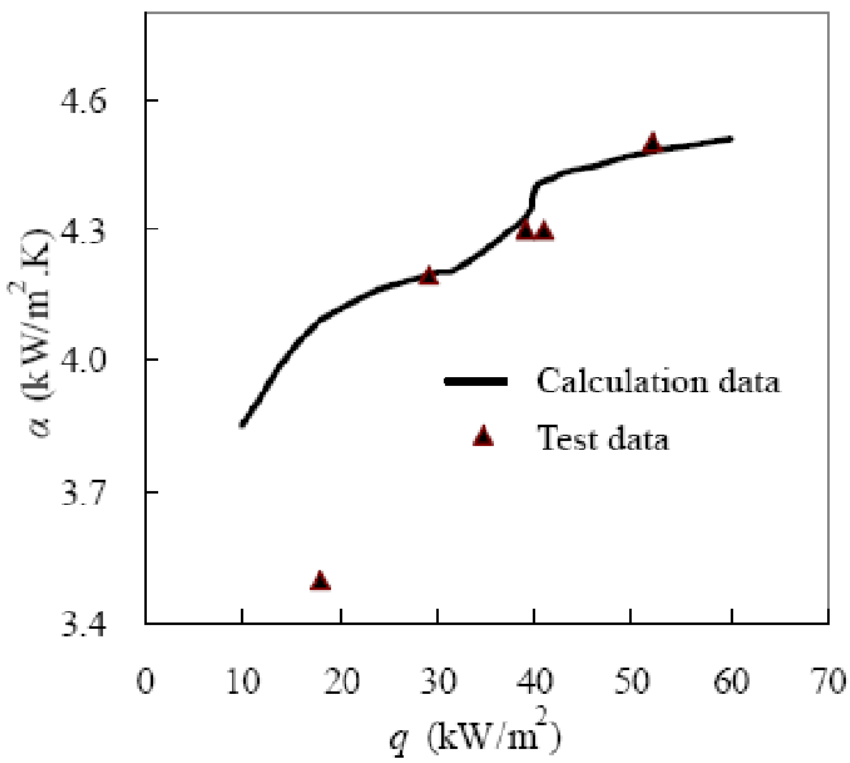

Fig. 2. Heat transfer coefficient varying with heat addition density 
Fig.2 indicates the heat transfer coefficient in evaporator section under different heat addition densities. With the increase of heat addition density signifying a higher total heat transfer quantity, the heat transfer coefficient in the evaporator section increases. As different from Figure 1, the relationship between the heat transfer coefficient and the heat addition density is non-linear. The calculation results fit the experimental results better under the higher heat addition densities $\left(40 \mathrm{~kW} / \mathrm{m}^{2}<q<60 \mathrm{~kW} / \mathrm{m}^{2}\right)$ as opposed to the lower heat values $\left(10 \mathrm{~kW} / \mathrm{m}^{2}<q<38 \mathrm{~kW} / \mathrm{m}^{2}\right)$. Within the experimental range of heat addition densities, the heat transfer coefficient for the evaporator section of the thermosyphon could reach as high as $4.6 \mathrm{~kW} / \mathrm{m}^{2} . \mathrm{K}$.

Using the particular numerical methodologies adopted here, one can gain additional relevant information for the heat transfer and two-phase flow details in the thermosyphon which generally are not accessible by experimental means. Fig.3 depicts the axial velocity distribution of the vapor phase under different heat addition densities.

With the increase of heat addition density, the axial velocity of the vapor increases. For a certain heat addition density value, the axial velocity increases gradually from zero in the evaporator section while it decreases gradually to zero in the condenser section and reaches a maximum value in the adiabatic section. The axial velocity of vapor could reach as high as $10 \mathrm{~m} / \mathrm{s}$ when the heat addition density is given as $1.97 \mathrm{~kW}$, a result predicted to cause substantial flow induced vibration and turbulence.

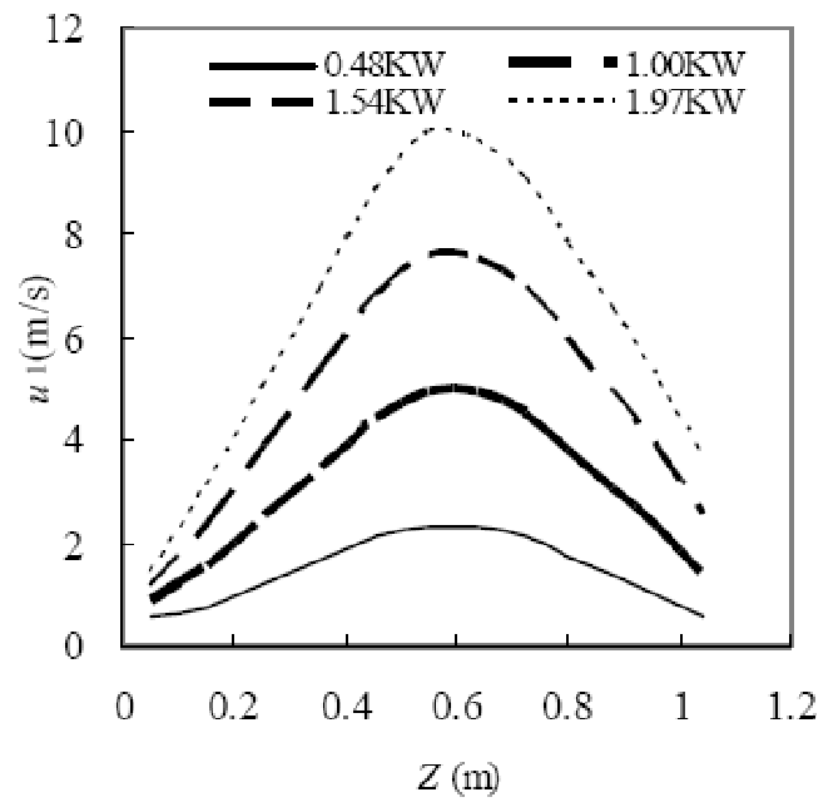

Fig. 3. Axial velocity distribution of vapor phase varying with heat addition density 


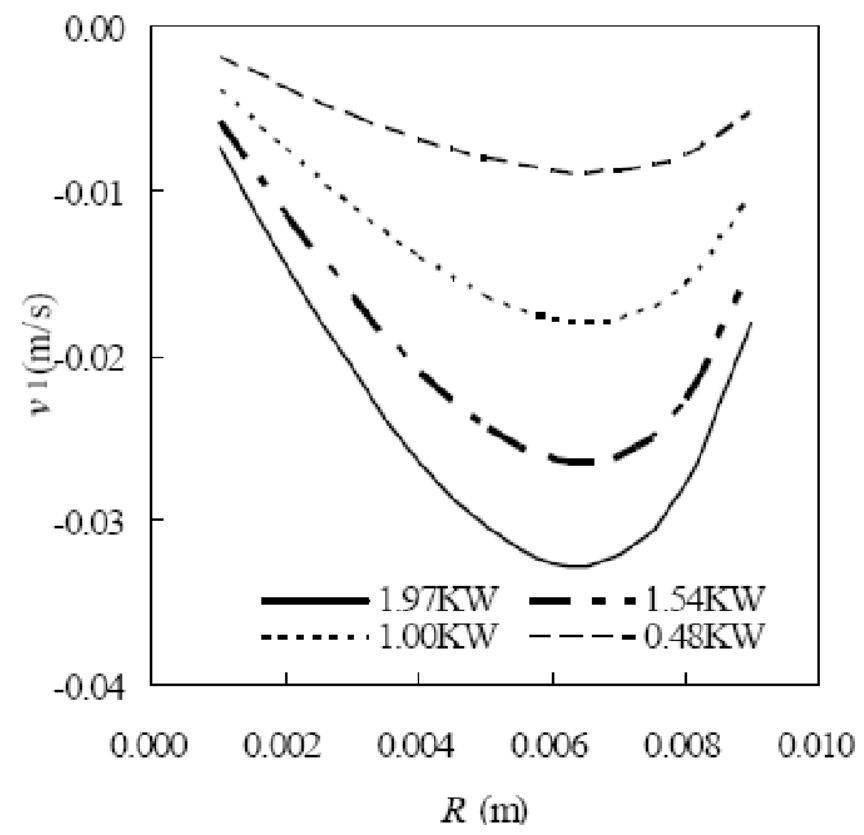

Fig. 4. Radial velocity distribution of vapor phase in evaporator section varying with heat addition density

Fig.4 indicates the radial velocity distribution for the vapor phase in the evaporator section under different heat addition densities. In this particular set of circumstances, the radial velocity of the vapor from the center initially increases from zero and there exists a maximum radial velocity. The radial velocity values for the vapor increase with the increase of the heat addition density. From Figures 3 and 4, one can draw the conclusion that axial velocity of vapor is considerably larger than the radial component ; however, since the radial velocity displays a larger variation vis a vis the axial, here it is shown conclusively that the flow field details in a typical thermosyphon are indeed very complex and numerical simulations henceforth provides for more detailed design and development information.

\section{Conclusions}

In this paper models to numerically simulate the heat transfer and two-phase flow details within a certain thermosyphon design are presented. Based on the widely employed SIMPLE and IPSA algorithms, successful computations highlighting the inherent operational characteristics of a typical design are carried out. Specifically, relevant flow and heat transfer details are revealed as influenced by the external operational working conditions. Furthermore, the celebrated IPSA algorithm as employed in the current effort strictly indicates that it can profitably and effectively be used to solve two phase flow problems including phase change. 
The predicted results agree well on the whole regarding the influence of operational parameters on the performance of the particular thermosyphon geometry. The linear relationship between the average temperature in the evaporator section and the heat addition density is conclusively borne out while the relationship involving the heat transfer coefficient and the heat addition density turns out to be highly nonlinear. Within the experimental range of heat addition densities, the heat transfer coefficient for the evaporator section of the thermosyphon could reach as high as $4.6 \mathrm{~kW} / \mathrm{m} 2 . \mathrm{K}$. The axial velocity of the vapor increases with the increase of heat addition density. The axial velocity of the vapor is substantially larger than the radial component; however, since the radial velocity variation is shown to be substantial vis a vis the axial, here it is shown conclusively the flow details in a typical thermosyphon are indeed extremely complex and hence appropriate numerical simulations provide vital design and development information seriously lacking for innovative hardware design exercises.

Since only a limited number of numerical simulation studies on the internal vaporliquid two-phase flow and heat transfer details in heat pipes and thermosyphons have been published [11-15], the numerical methodologies employed and successfully used in this paper can be used as a basis for further studies and hopefully in designing reliable hardware development correlations.

Acknowledgments. This work is financially supported by the Qing-Lan Project of Jiangsu Province for universities' outstanding youth skeleton teachers under contract No.161220605 and the Startup-grant of Jiangsu University of Science and Technology for academic research under contract No.2005JD009J. The corresponding author also owes great thanks to School of Mechanical, Aerospace and Civil Engineering in the University of Manchester for providing excellent collaborative research facilities.

\section{References}

1. Sauciuc, B.: Design and Testing of the Super Fiber Heat Pipes for Electronics Cooling Applications. In: Annual IEEE Semiconductor Thermal Measurement and Management Symposium, pp. 27-32. IEEE Press, New York (2001)

2. Sathe, A.: Review of Recent Developments in Some Practical Aspects of Air-cooled Electronic Packages. Journal of Heat Transfer, Transactions ASME 120, 830-838 (1998)

3. Ryan, J.M., Roshan, J., Song, L.: Integrated Thermal Management Techniques for High Power Electronic Devices. Applied Thermal Engineering 24, 1143-1156 (2004)

4. Zuo, Z.J., North, M.T., Wert, K.L.: High Heat Flux Heat Pipe Mechanism for Cooling of Electronics. IEEE Transactions on Components and Packaging Technologies 24, 220-225 (2001)

5. Lahey, R.: The Analysis of Phase Separation and Phase Distribution Phenomena Using Two-fluid Models. Nucl.Engng. Des. 122, 17-25 (1990)

6. Ishii., M.: Thermo-fluid Dynamic Theory of Two-phase Flow. Eyrolles Book Publishing House (1975)

7. Spalding, D.B., Markatos, N.C.: Computer Simulation of Multi-Phase Flows. CFDU. Imperial College UK (1983)

8. Shiraishi, M., Kikuchi, K.: Investigation of Heat Transfer Characteristics of Two-phase Closed Thermosyphon. In: Advances in Heat Pipe Technology, pp. 95-104 (1981) 
9. Kaya, T., Goldak, J.: Three Dimensional Numerical Analysis of Heat and Mass Transfer in Heat Pipes. Heat And Mass Transfer 43, 775-785 (2007)

10. Xia, J.L., Xin, M.D.: Heat Transfer Enhancement with Metal Powder on the Inner Wall of a Certain Two Phase Closed Thermosyphon. Journal of Chongqing University, 32-37 (1984)

11. Ghajar, M., Darabi, J.: Numerical Modeling of Evaporator Surface Temperature of a Micro Loop Heat Pipe at Steady State Condition. Journal of Micromechanics and Microengineering 15, 1963-1971 (2005)

12. Chen, M.M., Faghri, A.: An Analysis of The Vapor Flow and The Heat Conduction Through the Liquid-wick and Pipe Wall In a Heat Pipe With Single or Multiple Heat Sources. International Journal of Heat and Mass Transfer 33, 1945-1955 (1990)

13. Schmalhofer, J., Faghri, A.: A Study of Circumferentially Heated and Block-heated Heat Pipes-II. Three-dimensional Modeling as a Conjugate Problem. International Journal of Heat and Mass Transfer 36, 213-226 (1993)

14. Zhu, N., Vafai, K.: Analysis of Cylindrical Heat Pipes Incorporating the Effects of Liquidvapor Coupling and Non-Darcian Transport - a Closed Form Solution. International Journal of Heat and Mass Transfer 42, 3405-3418 (1999)

15. Chernysheva, M.A., Maydanik, Y.F.: Numerical Simulation of Transient Heat and Mass Transfer in a Cylindrical Evaporator of a Loop Heat Pipe. International Journal of Heat and Mass Transfer 51, 4204-4215 (2008) 